\title{
Two Young Patients with Stroke in Conjunction with Migraineus Headache
}

\author{
Xue Ming ${ }^{*}, 1$, Hussam Yacoub $^{1}$, Anna Khanna ${ }^{2}$ and George C. Wagner ${ }^{3}$ \\ ${ }^{1}$ Department of Neurosciences and Neurology, UMDNJ-New Jersey Medical School, Newark, NJ, USA \\ ${ }^{2}$ Neurological Associates, Ocala Regional and West Marion Medical Center, Ocala, FL, USA \\ ${ }^{3}$ Department of Psychology, Rutgers University, New Brunswick, NJ, USA
}

\begin{abstract}
In adults, migraine with aura is considered to be a risk factor for stroke, especially when other risk factors exist. However, the relationship between migraine with aura and risk of cerebral infarct in children has not been well established and requires further study. We describe four young patients who suffered from migraine with aura and focal neurological deficits. All four patients had migraine with aura and focal neurological deficits associated with headaches. Two of the four young patients had cerebral infarct during their migraine headaches. Detailed investigation did not reveal the etiology of the cerebral infarct in these two adolescents. No risk factor for stroke was apparent in any of the four patients and no specific risk factor or reliable clinical feature could be used to identify these young patients at risk for irreversible cerebral ischemia. Thus, our observations provide support that there may be an association between migraine with aura and risk of stroke in children. We, therefore, advocate aggressive treatment using prophylactic agents to prevent migraine attacks in children suffering from migraine with aura, especially when auras are prolonged.
\end{abstract}

Keywords: Migraine with aura, children, risk factors, stroke, vasospasm.

\section{INTRODUCTION}

According to the International Headache Society, migraine may be classified into a number of categories among which are migraine with aura and migrainous infarct [1]. Auras include focal neurological deficits such as reversible visual disturbances, sensory symptoms, and/or dysphasic speech. In addition, hemiplegic symptoms are considered to be a migraine aura, though migraine with hemiplegia may be coded separately [1]. In any case, when making a diagnosis of migraine with aura, it is important to note that the headache itself may occur simultaneously with or follow the aura and that the neurological deficits usually resolve within 60 minutes and only rarely persist longer than 24 hours [1].

In contrast to migraine with aura, permanent neurological sequelae associated with attacks of migraine were described by Charcot as early as the $19^{\text {th }}$ century and were originally referred to as complicated migraine [2]. This form of migraine, now termed migrainous infarct, accounts for between 0.5 and $1.5 \%$ of all strokes [3]. The prevalence of active migraine during acute ischemic stroke in the Lusanne stroke registry was reported to be as high as $3.7 \%$ for all patients, $15.8 \%$ for patients under the age of 45 , and $74 \%$ for women under 45 years of age [4]. In addition, Henrich and Horwitz reported a migraine prevalence of $19 \%$ in patients with ischemic stroke under the age of 65 [5]. Furthermore, it has been suggested that migraine with aura is a risk factor for ischemic stroke [6-9] with approximately a 2 -fold increased

*Address correspondence to this author at the Department of Neurosciences and Neurology, UMDNJ-New Jersey Medical School, 90 Bergen Street, DOC 8100, Newark, NJ 07103, USA; Tel: 1- 973-972-5204; Fax: 1-973972-9553; E-mail: mingxu@umdnj.edu risk in women having migraine with aura as compared to women with no history of migraine [10]. An increased frequency of white matter hyper-intensities seen on magnetic resonance imaging (MRI) of the brain have also been reported in patients with migraine, an observation consistent with a history of frequent ischemia $[11,12]$. A recent study showed an apparent association between migraine frequency and cardiovascular disease including ischemic stroke in women suffering from migraine with aura. Ischemic stroke was associated with high frequency (weekly) migraine attacks [13]. Taken together, these studies show a significant association between migraine with aura and ischemic stroke, especially in younger patients and in women.

The diagnosis of migraine in children and adolescents is more complex than in adults primarily because they often lack typical features. In general, migraine with aura is thought to be less prevalent in children with migrainous infarct even less commonly observed [14]. A number of epidemiological studies evaluating the risk of ischemic stroke in migraineurs included adolescents in their study populations [5,15-22]. These studies demonstrated that migraine with aura was indeed a risk factor for ischemic stroke, especially in the presence of other stroke risk factors including contraceptive use, hypertension, and diabetes. However, these epidemiological studies on risk factors for migrainous stroke included predominantly adult subjects, with only a small number of adolescent participants. Currently, little is known about the risk of migrainous stroke in populations consisting exclusively of children and adolescents.

While it is clear that migraine with aura is a risk factor for stroke in adults, such a relationship has not been adequately established in children. In this case series review, we 
present the clinical characteristics of four adolescents who exhibited migraine with focal neurological deficits, with or without radiographic ischemic infarcts, along with a brief review and discussion on the pathogenesis of migrainous infarction. With these observations, we suggest prophylactic migraine treatment for children suffering from migraine with aura.

This study was approved by the Institutional Review Board of our institute and informed consent procedure was not applicable to this retrospective review without personal identifiers.

\section{RESULTS}

\section{Case 1}

A 16 year-old non-obese Hispanic girl with approximately a seven-week history of migraine headache presented to our emergency department in the early summer of 2000 with a sudden onset of left hemiparesis. Her previous migraine episodes were accompanied by nausea and photophobia but were neither associated with aura nor complicated by neurological deficits. There was no family history of migraine or stroke. She did not smoke and had never used oral contraceptives, hormonal treatment or illicit drugs. She had been otherwise healthy. Earlier on the day of presentation, the patient experienced an aura of tingling in her left hand that rapidly progressed to involve her left face and leg. The patient subsequently developed a complete left hemiplegia. One hour after the onset of symptoms, the patient was found to also have a complete left hemisensory loss. Her symptoms resolved completely as she was undergoing a head computed tomography (CT) scan and CT angiography, both of which were normal. There was no evidence of cerebral vasculopathy. Following the CT angiography the patient developed another episode of transient left hemiplegia that rapidly improved but left a residual mild pronator drift. Fluidattenuated inversion recovery MRI of the brain was performed two and a half hours after onset of her initial symptoms and revealed increased signal intensity in the right basal ganglia extending into the posterior limb of the right internal capsule, most consistent with an acute ischemic infarct (Fig. 1). Diffusion-weight MRI and apparent diffusion coefficient confirmed the presence of an ischemic infarct (images not shown). The patient was started on intravenous heparin to maintain a partial thromboplastin time of $60-80$ seconds. Evaluation for a possible coagulopathy prior to initiation of heparin, inborn error of metabolism and mitochondrial disorders was unremarkable. A trans-esophageal echocardiogram revealed no evidence of a patent foramen ovale, thrombus, or other structural abnormality. Approximately six hours after the onset of hemiplegia, the patient developed a right temporal pulsatile headache associated with left arm tingling which rapidly resolved. Migrainous infarction was considered and the possibility of vasospasm responsible for the reversible sensory deficit was raised. The patient was started on nimodipine 30mg via parental route every 6 hours for 48 hours followed by verapamil $80 \mathrm{mg}$ daily for presumed cerebral vasospasm. The doses of nimodipine and verapamil were carefully titrated to avoid hypotension. By the following day, her headache and left pronator drift had resolved completely. After her discharge, the patient continued to have episodic headaches with left arm numbness that were often triggered by salty and greasy food. The diagnosis of migraine with aura was thus made. The verapamil dose was increased to $120 \mathrm{mg}$ daily and the patient was advised to refrain from any offensive food. In addition, valproic acid was added to the patient's migraine prophylaxis regimen and she has remained headache free and has no additional neurological deficits for at least four years of follow-up.

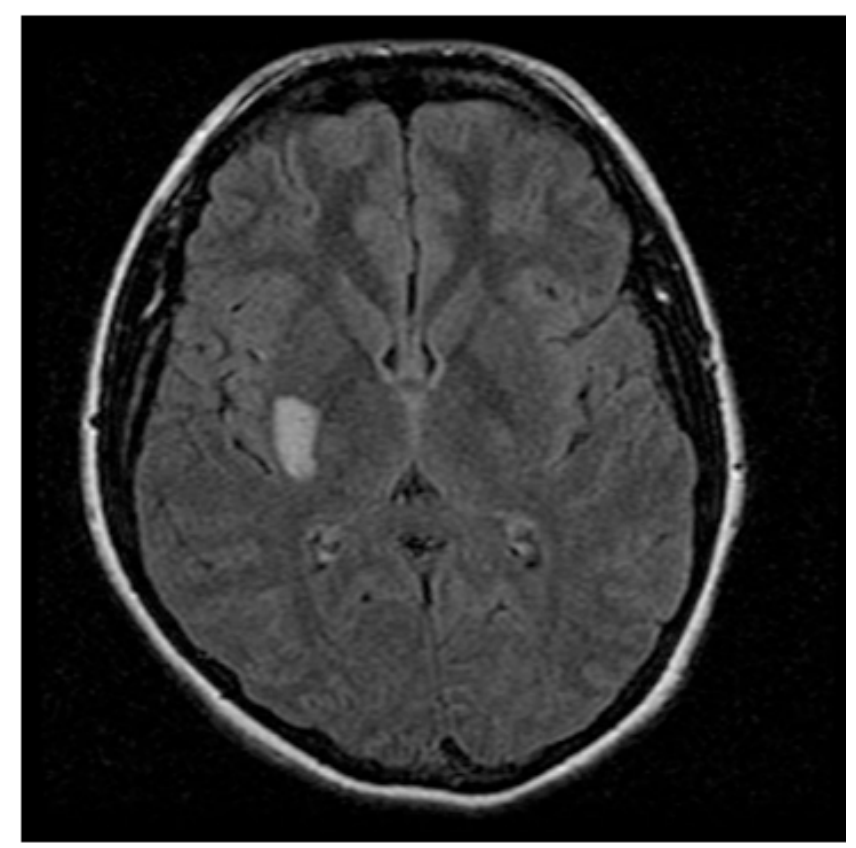

Fig. (1). Fluid-attenuated inversion recovery (FLAIR) MRI showed a hyper-intensity signal in the right basal ganglia extending to the posterior limb of the right internal capsule.

\section{Case 2}

A 12 year-old thin Filipino boy with a prior history of migraine headache presented to our emergency department with left-sided weakness. Three months prior to this presentation, the patient developed his first throbbing headache which was preceded by an aura of right facial throbbing sensation. He had nausea and photosensitivity during the headache. One day prior to his presentation, the patient reported an episode of headache associated with left-sided numbness. When he arrived to our emergency department, his symptoms had progressed to left hemiparesis. On examination, the patient was found to have left hemiparesis and left hemisensory loss to pain and temperature. Initial cranial CT was unremarkable. MRI of the brain performed 48 hours after onset of symptoms showed an ischemic infarct in the posterior limb of the right internal capsule (images not shown). Cerebral magnetic resonance angiography (MRA) was unremarkable with no evidence of cerebral angiitis. Evaluation for coagulopathy, inborn error of metabolism and DNA marker for mitochondrial encephalomyopathy, lactic acidosis, and stroke-like episodes was unremarkable. Cerebral spinal fluid (CSF) analysis showed no leukocytosis. The patient had a first-degree relative who experienced migraine without aura, but there was no family history of genetic or cerebral vascular disorders. An echocardiogram showed no structural abnormality, thrombus, or patent foramen ovale. Over the next four days of hospitalization, the patient's 
symptoms improved with a residual mild decreased fluidity in left rapid alternating hand movement and left facial weakness. The patient received nimodipine $10 \mathrm{mg}$ intravenously every 8 hours for 72 hours (the dose was carefully titrated to avoid hypotension). The patient was discharged on aspirin $81 \mathrm{mg}$ daily. At the follow up visit, the patient reported to have infrequent migraine headaches with and without transient left sided face and arm numbness.

\section{Case 3}

A 15 year-old African American non-obese girl presented to our clinic with a two-year history of frequent migraine headache (once to twice per week). Approximately 6 months after her initial migraine onset, the patient experienced an aura of left hemi-sensory numbness shortly prior to her headache and lasted a few hours after her headache resolved. Since then, the majority of her migraine headache episodes had been associated with either right or left hemi-sensory numbness lasting less than 60 minutes. Her headache was pulsatile and associated with photophobia. Physical examination during one of the headache episodes showed reduced sensation to pain and temperature on the left side of her body. MRI and MRA of the brain performed during a typical headache episode, when the headache had just resolved but hemi-sensory loss still persisted, showed no abnormality. There was no evidence of cerebral vasculitis. Evaluation for coagulopathy and risk factors for stroke were unremarkable. The patient reported that she had never smoked and had never been on oral contraceptives or used any recreational drugs. There was no family history of migraine or stroke. The patient's migraine frequency and duration of headache episodes improved with the addition of valproate to her existing verapamil regimen. Despite having occasional headache with aura, the patient had no neurological deficits during multiple clinical evaluations the following 18 months. The diagnosis of migraine with aura was made when the duration of aura became typical and the evaluation excludes other causes of her fully-reversible sensory symptoms.

\section{Case 4}

A 20 year-old Hispanic non-obese man was evaluated in our clinic for a 5-year history of migraine with aura. There was no family history of migraine headache or stroke. He denied cigarette smoking and recreational drug use. His headaches started when he was 15 years old. The headaches were always preceded by a bilateral peripheral visual field loss with only a small tunnel of central vision preserved. The visual disturbance aura typically began 10-15 minutes prior to the headache and resolved once the headache began. The patient's headache did not respond to propranolol. In addition, the patient reported two prior episodes of headache that was preceded by right hemiplegia and resolved within 30 minutes after headache onset. The patient's headache was triggered by eating greasy and salty food. An MRI of the brain performed during the inter-ictal phase 5 years after initial onset of migraine was unremarkable. The patient was started on verapamil with improvement of headache frequency. Over the period of one year after the initiation of verapamil therapy, the patient's headache became less frequent and there had been no associated focal neurological deficits. No abnormal physical exam findings of visual or motor deficits were appreciated during multiple subsequent clinical visits.

\section{DISCUSSION}

Each of the four cases of migraine with aura outlined above presented with unique and atypical features that resulted in a diagnostic challenge. The patient in Case 1, who previously had no aura associated with her migraine, presented with migrainous infarct resulting in focal deficit. The presence of aura during subsequent headache episodes resulted in reclassification of her condition as having migraine with aura. In contrast, the patient in Case 2 was quickly diagnosed with migrainous infarct due to the presence of previous migraine with aura, although the aura was atypical. Patients in both cases developed subcortical cerebral ischemic infarcts only months after the initial onset of migraine with resultant motor and sensory deficits. The patient presented in Case 3 had sensory deficits which lasted greater than 60 minutes but less than 7 days with no MRI evidence of an ischemic infarct. The long duration of her hemisensory loss, persisting for at least 10 hours after her headache resolved, suggests a prolonged but reversible vasospasm resulting in recurrent transient ischemia. However, her headache did not fulfill the International Headache Society diagnostic criteria for either migraine with aura or persistent aura without infarct. Finally, case 4 fulfilled the diagnosis of sporadic hemiplegic migraine with aura, presenting with visual loss or hemiplegia associated with headache. Similar to Case 3, there was no clinical or MRI evidence of ischemic infarct.

Two of the four cases presented had stroke. A full review of the history failed to reveal risk factors for stroke in any of the patients. In all cases, age, sex, ethnic background or family history was not a risk factor in determining a permanent infarct. Additionally, none of the patients used hormonal or other agents associated with infarct or coagulopathy and none of the children smoked cigarettes. Evaluation for mitochondrial disorders, cerebral vasculopathy, the syndrome of transient headache and neurologic deficits with CSF lymphocytosis did not reveal any explanation for the neurological deficits in these cases.

Two of the four adolescents who suffered from migraine and focal neurological deficits had permanent ischemic infarcts. Extensive investigation in these two children failed to reveal an etiology of the ischemic infarct. Thus, no features were available prior to the actual stroke that allowed predictive insight into the future neurological deficits.

The exact mechanism underlying migrainous infarct is yet to be understood. However, a dysfunction of cerebral arteries during migraine attacks and findings of silent infarctlike brain lesions in migraineurs imply an association between stroke and migraine. Possible mechanisms leading to the infarct include vasospasm and decrease in cerebral blood flow, vasculopathy (such as endothelial dysfunction, genetic vasculopathies, and polymorphisms in genes regulating endothelial function), platelet hyper-aggregability, and associations with cardiac abnormalities [6, 23-26]. Previous studies utilizing cerebral angiography in patients with neurological deficits during a migraine attack demonstrated carotid or vertebral artery system vasospasm to a degree that might lead to cerebral hypoperfusion and infarct [27, 28]. Further- 
more, it has been theorized that cerebrovascular dysautonomia may occur with transient vasospasms and periods of vasodilation $[27,29]$. Linetsky et al. prospectively studied six patients with stroke who had an ischemic lesion in the same vascular distribution as their migraine aura and found that stroke occurred during the ictal phase of the migraine [28]. In their study, patients underwent a breath-holding test during continuous transcranial doppler monitoring. They hypothesized that carbon dioxide accumulation would cause vasoconstriction and a resultant increase in velocity in cerebral blood vessels. After 25-40 seconds of breath-holding, the middle cerebral artery velocities remained unchanged on the affected side but increased by $35 \%-45 \%$ on the unaffected side. This supports the idea that during a migraine attack, the affected cerebral vessels become maximally dilated. Similarly, Rosengarten et al. demonstrated increased cerebrovascular reactivity (an increased tone of the vessels) in adolescents with migraine without aura during migraine attacks as compared to that during headache-free intervals [30]. Collectively, these two studies support the theory that vascular dysautonomia plays a central role in the pathogenesis of cerebral infarcts associated with migraine.

There is increasing evidence that cortical spreading depression may be responsible for the aura associated with migraine. Cortical spreading depression occurs when a brief but intense neuronal and glial depolarization spreads across the cerebral cortex followed by prolonged neuronal suppression [31-33]. Neuroimaging studies [34-36] have revealed changes in visual cortex blood flow during migraine aura that are consistent with those reported in rats during cortical spreading depression [32]. In rats, cortical spreading depression results in a persistent increase in oxygen consumption with impaired neurovascular and neurometabolic coupling [37] ultimately leading to glucose depletion [38]. This cortical spreading depression-induced oligemia usually begins in the occipital lobes and progresses anteriorly and is thought to be responsible for migraine aura [39]. In fact, the consistent occurrence of cortical spreading depression together with oligemia in the occipital lobes might help explain the predominance of visual disturbance during migraine-associated auras. Additionally, platelet microaggregation (an abnormality that can be augmented by oral contraceptives, alcohol, cigarette smoking or diabetes) was more likely to be associated with "spreading oligemia" in migraineurs as compared to controls [39]. Thus, it is postulated that cortical spreading depression is associated with a vascular dysautonomia (vasodilation, hyperemia) and is followed by a period of oligemia [40, 41].

The exact relationship between cortical spreading depression and ischemic infarct has been the topic of a number of excellent reviews [42-44]. It is clear that cortical spreading depression together with peri-infarct depolarization follows cerebral artery infarct and may be partly responsible for increasing the extent of neuronal damage [45,46]. It is also likely that cortical spreading depression will trigger infarct if it is sufficiently protracted, leading to compromised tissue metabolism [see 43]. Case 1 appears to represent the former scenario with the headache occurring hours after the ischemic infarct. Case 2 appears to represent the second scenario with the headache and aura clearly proceeding the ischemic infarct.
The factors that might determine if a focal neurological deficit was a reversible aura or permanent infarct have yet to be fully evaluated. The International Headache Society commented that a decrease in regional cerebral blood flow, usually above an ischemic threshold, corresponds with the clinical aura seen in migraineurs [1]. We now speculate that this threshold might be lower in susceptible individuals. If this hypothesis is to be held, a prolonged aura associated with vascular dysautonomia might lead to cerebral ischemia and infarct. If the presumed diminished regional cerebral blood flow for case 2 was below the ischemic threshold, it might have resulted in an infarct. Alternatively, if the diminished regional blood flow for cases 3 and 4 was above the threshold, it might allow for reperfusion without permanent injury to the corresponding brain tissue. The four cases outlined previously demonstrate a range of neurological deficits that possibly reflect a spectrum of different degrees of vasospasm. The patient in case 1 sustained a cerebral infarction associated with her first migraine with aura that gradually improved with the combination of verapamil and valproate. In contrast, the patient in case 2 experienced aura without focal neurological deficits during his previous migraine attacks but permanent infarct occurred during his current presentation. It is possible that the degree and duration of cerebral vasospasm/hypoperfusion are the two variables that may predict a wide range of neurological deficits across patients with migraine and determine whether focal deficits are reversible or permanent. These two variables might help to explain the range of neurological deficits observed in our patients.

Drug therapy has been considered a precipitating factor of cerebral infarction in patients with a history of migraine. Although recent studies dispute triptans as causative agents of migraine stroke [47], the use of ergotamines and sumatriptan have been shown to cause constriction of cerebral vessels and precipitate ischemia $[12,48]$. Dash et al. described a case of cerebral infarction with angiographic evidence of vasospasm in a patient on sumatriptan for chronic migraine headache [49]. Angiographic follow up revealed a significant improvement in cerebral vasospasms seven days after discontinuing the sumatriptan. Likewise, the use of propranolol as a prophylactic agent for migraine has also been suggested as a contributing factor for hypotension and cerebral hypoperfusion leading to cerebral infarction [50, 51]. None of our patients used an ergotamine or a beta blocker, thus ruling out these drugs as causing the auras and neurological deficits.

The cases outlined herein demonstrate that no specific risk factor or reliable clinical marker could be used to identify children at risk for irreversible cerebral ischemia. Indeed, migraine with aura was present in all four cases, and two showed permanent infarct, an observation that provides support linking migraine with aura to stroke in children. We acknowledge that this case series does not have the rigor of a large cohort prospective study on the association of migraine with aura and stroke in children. To date, this association has not been addressed in children, though it has been established in adults.

In summary, we conclude that there may be a connection of migraine with aura and stroke in children and adolescents. Although it is not clear whether prophylaxis can prevent 
stroke, our case series showed benefit of the prophylactic treatment in reducing frequency of migraine attacks thereby theoretically reducing the chance of stroke, especially since high frequency migraine attack was associated with ischemic stroke in adults [13]. Until a reliable clinical biomarker can be identified and given that prophylactic therapy is relatively safe but seriously underutilized, we advocate aggressive treatment using prophylactic agents that can prevent vasospasm in children who suffer from migraine with aura, especially for prolonged auras. We also caution against the use of any agent including triptans that can lead to vasospasm in children with migraine [52].

\section{ACKNOWLEDGEMENT}

This work was performed without external funding at UMDNJ-New Jersey Medical School, Newark, NJ. All authors disclose no financial conflict or interest in this study. The authors wish to acknowledge John Lincoln MD for his critical review of this manuscript.

\section{ABBREVIATIONS}

\section{CT $=$ Computerized Tomography \\ MRI = Magnetic Resonance Images \\ MRA = Magnetic Resonance Angiography \\ REFERENCES}

[1] Headache Classification Subcommittee of the International Headache Society. The international classification of headache disorders, $2^{\text {nd }}$ Edition. Cephalalgia 2004; 24: 24-49.

[2] Charcot JM. Sur un cas de migraine opthalmoplegique. Prog Med 1890; 1: 83-4.

[3] Arboix A, Massons J, Garcia-Erdes L. Migrainous cerebral infarction in the Sagrat Cor Hospital of Barcelona stroke registry. Cephalalgia 2003; 23: 389-94.

[4] Milhaud D, Bogousslavsky J, van Melle G, et al. Ischemic stroke and active migraine. Neurology 2001; 57: 1805-11.

[5] Henrich JB, Horwitz RI. A controlled study of ischemic stroke risk in migraine patients. J Clin Epidemiol 1989; 42: 773-80.

[6] Etminan M, Takkouche B, Isorna C, et al. Risk of ischaemic stroke in people with migraine: systematic review and meta-analysis of observational studies. BMJ 2005; 330: 63-5.

[7] Kurth T, Slomke MA, Kase CS, et al. Migraine, headache, and the risk of stroke in women: a prospective study. Neurology 2005; 64 : $1020-6$.

[8] Stang PE, Carson AP, Rose KM, et al. Headache, cerebrovascular symptoms, and stroke: the Atherosclerosis Risk in Communities Study. Neurology 2005; 64: 1573-7.

[9] Scher AI, Terwindt GM, Picavet HS, et al. Cardiovascular risk factors and migraine: the GEM population-based study. Neurology 2005; 64: 614-20

[10] Wingerchuk DM , Spencer B, Dodick DW, et al. Migraine with aura is a risk factor for cardiovascular and cerebrovascular disease. A Critically Appraised Topic. Neurologist 2007; 13: 231-3.

[11] Kruit MC, Launer LJ, Ferrari MD, et al. Infarcts in the posterior circulation territory in migraine. The population-based MRI CAMERA study. Brain 2005; 128: 2068-77.

[12] Kruit MC, van Buchem MA, Hofman PA, et al. Migraine as a risk factor for subclinical brain lesions. JAMA 2004; 291: 427-34.

[13] Kurth T, Schurks M, Logroscino G, et al. Migraine frequency and risk of cardiovascular disease in women. Neurology 2009; 73: 5818 .

[14] Bigal ME, Lipton RB. Migraine at all ages. Curr Pain Headache Rep 2006; 10: 207-13.

[15] Carolei A, Marini C, De Matteis G. History of migraine and the risk of cerebral ischemia in young adults. Lancet 1996; 347: 15036.

[16] Collaborative Group of the Study of Stroke in Young Women. Oral contraceptives and stroke in young women. JAMA 1975; 1231: $718-22$.
[17] Haapaniemi H, Hillbom M, Juvela S. Life-style associated risk factors for acute brain infarction among persons of working age. Stroke 1997; 28: 26-30.

[18] Lidegaard O. Oral contraceptives, pregnancy and the risk of cerebral thromboembolism: the influence of diabetes, hypertension, migraine and previous thrombotic disease. Br J Obstet Gynaecol 1995;102: 153-9.

[19] Marini C, Carolei A, Roberts RS, et al. Focal cerebral ischemia in young adults: a collaborative case-control study. Neuroepidemiology $1993 ; 12: 70-81$.

[20] MacClellan LR, Giles W, Cole J, et al. Probable migraine with visual aura and risk of ischemic stroke: the Stroke Prevention in Young Women Study. Stroke 2007; 38: 2438-45.

[21] Nightingale AL, Farmer RD. Ischemic stroke in young women: a nested case-control study using the UK general practice research database. Stroke 2004; 35: 1574-8.

[22] Tzourio C, Iglesias S, Hubert JB, et al. Migraine and risk of ischemic stroke: a case-control study. BMJ 1993; 307: 289-92.

[23] Bousser MG, Welch KMA. Relation between migraine and stroke. Lancet Neurol 2005; 4: 533-42.

[24] Tietjen GE, Herial NA, White L, et al. Migraine and biomarkers of endothelial activation in young women. Stroke 2009; 40: 2977-82.

[25] Macclellan LR, Howard TD, Cole JW, et al. Relation of candidate genes that encode for endothelial function to migraine and stroke. The stroke prevention in young women study. Stroke 2009; 40: e550-7.

[26] Stam AH, Haan J, van Maagdenberg AMJM, et al. Migraine and genetic and acquired vasculopathies. Cephalalgia 2009; 29: 100617.

[27] Sanin LC, Mathew NT. Severe diffuse intracranial vasospasm as a cause of extensive migrainous cerebral infarction. Cephalalgia 1993; 13: 289-92.

[28] Linetsky E, Leker RR, Ben-Hur T. Headache characteristics in stroke patients after migranous stroke. Neurology 2001; 7: 130-2.

[29] Rothrock JF, Walicke P, Swenson MR. Migrainous stroke. Arch Neurol 1988; 45: 63-7.

[30] Rosengarten MD, Sperner J, Görgen-Pauly U, et al. Cerebrovascular Reactivity in Adolescents With Migraine and Tension-Type Headache During Headache-Free Interval and Attack. Headache 2003; 43: 458-63.

[31] Leao APP. Spreading depression of activity in the cerebral cortex. J Neurophysiol 1944; 7: 359-90

[32] Lauritzen M. Pathophysiology of the migraine aura. The spreading depression theory. Brain 1994; 117: 199-210.

[33] Gorji A, Scheller D, Straub H, et al. Spreading depression in human neocortical slices. Brain Res 2001; 906: 74-83.

[34] Hadjikhani N, Sanchex del Rio M, Wu O, et al. Mechanisms of migraine aura revealed by functional MRI in human visual cortex. Proc Natl Acad Sci USA 2001; 98: 4687-92.

[35] Woods RP, Iacoboni M, Mazziotta JC. Brief report: bilateral spreading cerebral hypoperfusion during spontaneous migraine headache. N Engl J Med 1994; 331: 1689-92.

[36] Cutrer FM, Sorensen AG, Weisskoff RM, et al. Perfusion-weighted imaging defects during spontaneous migrainous aura. Ann Neurol 1998; 43: 25-31.

[37] Piilgaard H, Lauritzen M. Persistent increase in oxygen consumption and impaired neurovascular coupling after spreading depression in rat neocortex. J Cereb Blood Flow Metab 2009; 29: 1517 27.

[38] Hashemi P, Bhatia R, Nakamura H, et al. Persisting depletion of brain glucose following cortical spreading depression, despite apparent hyperaemia: evidence for risk of an adverse effect of Lea o's spreading depression. J Cereb Blood Flow Metab 2009; 29: 166-75.

[39] Welch KM. Migraine, a bio-behavioral disorder. Arch Neurol 1987; 44: 323-7.

[40] Cao Y, Welch KM, Aurora S, et al. Functional MRI-BOLD of visually triggered headache in patients with migraine. Arch Neurol 1999; 56: 548-54.

[41] Friberg L, Olesen J, Lassen NA, et al. Cerebral oxygen extraction, oxygen consumption, and regional cerebral blood flow during the aura phase of migraine. Stroke 1994; 43: 25-31.

[42] Moskowitz MA. Pathophysiology of headache - past and present Headache 2007; 47 (S1): S58-S63. 
[43] Del Zotto E, Pezzini A, Giossi A, et al. Migraine and ischemic stroke: a debated question. J Cereb Blood Flow Metab 2008; 28: $1399-421$.

[44] Foroozan R, Cutrer FM. Transient neurologic dysfunction in migraine. Neurol Clin 2009; 27: 361-78.

[45] Dohmen C, Sakowitz OW, Fabricius M, et al. Spreading depolarizations occur in human ischemic stroke with high incidence. Ann Neurol 2008; 63: 720-8.

[46] Nakamura H, Strong AJ, Dohmen C, et al. Spreading depolarization cycle around and enlarge focal ischaemic brain lesions. Brain 2010; 133: 1994-2006.

[47] Velentgas P, Cole JA, Mo J. Severe vascular events in migraine patients. Headache 2004; 44: 642-51.

[48] Wammes-van der Heijden EA, Rahimtoola H, et al. Risk of ischemic complications related to the intensity of triptan and ergotamine use. Neurology 2006; 67: 1128-34.
[49] Dash S, Bogdanova O, Xavier A, et al. Cerebral vasospasm from sumatriptan. Neurology 2004; 63: 2128

[50] Baumel B. Migraine: a pharmacologic review with newer options and delivery modalities. Neurology 1994; 44: S13-S17.

[51] Bardwell A, Trott J. Stroke in migraine as a consequence of propranolol. Headache 1987; 27: 381-3.

[52] Roach ES, Golomb MR, Adams R et al. American Heart Association Stroke Council; Council on Cardiovascular Disease in the Young. Management of stroke in infants and children: a scientific statement from a Special Writing Group of the American Heart Association Stroke Council and the Council on Cardiovascular Disease in the Young. Stroke 2008; 39: 2644-91.

Received: March 22, 2010

Revised: September 20, 2010

Accepted: September 27, 2010

(C) Ming et al.; Licensee Bentham Open.

This is an open access article licensed under the terms of the Creative Commons Attribution Non-Commercial License (http://creativecommons.org/licenses/by-nc/3.0/) which permits unrestricted, non-commercial use, distribution and reproduction in any medium, provided the work is properly cited. 